\title{
Changing Patterns of Primary Treatment in Korean Men with Prostate Cancer Over 10 Years: A Nationwide Population Based Study
}

\author{
Jinsung Park, MD, $P h D^{1}$ \\ Beomseok Suh, MD, MPH² \\ Dong Wook Shin, MD, DrPH, MBA² \\ Jun Hyuk Hong, MD, PhD ${ }^{3}$ \\ Hanjong Ahn, MD, PhD 3
}

\section{${ }^{1}$ Department of Urology,}

Eulji University Hospital, Daejeon,

${ }^{2}$ Department of Family Medicine,

Seoul National University Hospital, Seoul,

${ }^{3}$ Department of Urology,

Asan Medical Center,

University of Ulsan College of Medicine,

Seoul, Korea

\author{
Correspondence: Hanjong Ahn, MD, PhD \\ Department of Urology, Asan Medical Center, \\ University of Ulsan College of Medicine, \\ 88 Olympic-ro 43-gil, Songpa-gu, Seoul 05505, \\ Korea \\ Tel: 82-2-3010-3733 \\ Fax: 82-2-477-8928 \\ E-mail: hjahn@amc.seoul.kr \\ Received June 15, 2015 \\ Accepted September 30, 2015 \\ Published Online October 20, 2015
}

\begin{abstract}
Purpose
We investigated changing patterns of primary treatment in Korean men with prostate cancer (PC) and impact of sociodemographic factors on treatment choice from a nationwide cohort over 10 years.
\end{abstract}

\section{Materials and Methods}

We conducted a cohort study of a $2 \%$ nationwide random sample of Korean National Health Insurance. A total of 1,382 patients who had undergone active treatments for newly diagnosed PC between 2003 and 2013 were included. Time trends in primary treatment of PC, including radical surgery, radiation therapy (RT), and androgen deprivation therapy (ADT) were analyzed.

\section{Results}

Total number of patients undergoing active treatments increased significantly (162\%). Surgery cases showed the most significant increase, from $22.4 \%$ in 2003 to $45.4 \%$ in 2013 , while the relative proportion of ADT showed a tendency to decrease from $60.3 \%$ in 2003 to $45.4 \%$ in 2013 , and the relative proportion of RT was variable over 10 years (from $7.2 \%$ to 18.4\%). While treatment patterns differed significantly according to age $(p<0.001)$ and income classes $(p=0.014)$, there were differences in primary treatment according to residential area. In multinomial logistic regression analysis, older patients showed significant association with ADT or RT compared to surgery, while patients with higher income showed significant association with surgery.

\section{Conclusion}

Treatment pattern in Korean PC patients has changed remarkably over the last 10 years. Sociodemographic factors do affect the primary treatment choice. Our results will be valuable in overviewing changing patterns of primary treatment in Korean PC patients and planning future health policy for PC.

\section{Introduction}

Prostate cancer (PC) is the most common male cancer in the United States, with 233,000 new cases in 2014, and is the second most common cause of cancer-related death [1]. In Korea, PC is currently the fourth most common male cancer,

\section{Key words}

Practice patterns, Prostatic neoplasms, Prostatectomy, Radiotherapy 
antigen (PSA) screening are likely to be implicated in such increase of PC in Korea.

Treatment options for PC vary depending on disease severity and patient characteristics including age, comorbidity, and personal preferences. In addition, sociodemographic characteristics, including race, place of residence, income level, and insurance status [4-6], as well as clinician factors [7], may play a role in treatment decisions for PC. PC is a highly prevalent disease and has a public health impact in western countries, therefore many studies regarding trends in treatment patterns, causes of death, and impact of sociodemographic factors on patient outcomes have been reported based on large population-based cohorts [5-7]. However, despite the aforementioned rapid increase of PC in Korea, only a few studies regarding treatment patterns of PC in Korean men on the basis of a nationwide representative database have been reported. Through a better understanding of treatment patterns in Korean PC patients, we will gain insight into the current status of PC in Korea. In this study, we investigated changing patterns of primary treatment in Korean men with PC and impact of sociodemographic factors on primary treatment choice from a nationwide cohort over 10 years.

\section{Materials and Methods}

\section{Data sources and study population}

Data were obtained from 2002 to 2013 from the Korean National Health Insurance (KNHI) Sharing Service provided by KNHI Center. These data contain claims data in $2 \%$ of the entire Korean population (i.e., more than 1,000,000 people) randomly selected after stratifying the entire population according to socioeconomic parameters (age, sex, residential area, income, etc.). The database includes detailed information regarding disease diagnosed, imaging and laboratory tests, treatments (both medical and surgical) and death outcomes (cause and time) as well as aforementioned socioeconomic parameters. The study protocol was approved by the Institutional Review Board of Eulji University Hospital and Asan Medical Center.

Data with the code C61, indicating PC according to the National Center for Health Statistics International Classification of Diseases, 10th edition (ICD-10), were screened. Among 4,219 men aged 20 or older with the code C61 during the study period, newly diagnosed PC patients only after 2002 were included in order to minimize confounding effects due to pre-diagnosed PC. Thus, patients with C61 code at $2002(n=135)$ were excluded. In addition, patients who did not undergo active treatments for PC $(n=2,702)$, including those under watchful waiting or active surveillance, were excluded because they were not properly identifiable from the KNHI claims data. Thus, a total of 1,382 patients who had undergone active treatments, including radical surgery, radiation therapy (RT), and androgen deprivation therapy (ADT), for newly diagnosed PC constituted the study population.

\section{Primary treatment and operational definition}

We analyzed treatment patterns in terms of primary treatment modality including surgery, RT, and ADT. We also assessed the administration and timing of additional treatment before and after primary treatment. Neoadjuvant therapy was defined as additional therapy before primary surgery and RT, while adjuvant and salvage therapy was defined as additional therapy within 4 months and after 4 months after surgery, respectively.

Surgery includes open/laparoscopic radical prostatectomy (RP; KNHI reimbursement code 'R3950' and 'R3960') and robot-assisted RP (RARP). Because RARP is not reimbursed by KNHI, it cannot be identified by treatment codes. Thus, RARP was operationally defined as the absence of a surgery code despite the presence of general anesthesia (code 'L1211') and postoperative pathologic examination code (code 'C5500' or 'C5504' or 'C5505' or 'C5508' or 'C5918' or 'C5919'). RT includes all types of RT including conformal and intensity-modulated RT. Although patients underwent additional therapy before and after surgery and RT, they were categorized in terms of their primary treatment modality.

Primary ADT includes both surgical (orchiectomy) and medical castration. Medical castration includes luteinizing hormone-releasing hormone (LHRH) agonist only, antiandrogen only, and combined androgen blockade (CAB). Because chemotherapy is administered to patients who progress after primary treatment and salvage therapy, it was not categorized as primary treatment.

\section{Variables and statistical analysis}

Patient sociodemographic parameters included age, income class, and residential area. Patient age at diagnosis was divided into four categories $(<49,50-64,65-74$, and $\geq 75$ years) [8]. Income class was divided into five categories, as provided by KNHI. Residential area was divided into three categories (metropolitan, urban, and suburban/rural) based on population density.

Descriptive statistics were used to characterize treatment patterns according to sociodemographic factors. Pearson chisquare tests were used to describe the relationship between 
Table 1. Characteristics of the study population

\begin{tabular}{|c|c|c|c|c|}
\hline Variable & All subjects $(\mathrm{n}=1,382)$ & Surgery $(n=536,38.8 \%)$ & ADT $(n=669,48.4 \%)$ & RT (n=177, 12.8\%) \\
\hline \multicolumn{5}{|l|}{ Age (yr) } \\
\hline $20-49$ & $83(6.0)$ & $49(59.0)$ & $21(25.3)$ & $13(15.7)$ \\
\hline $50-64$ & $626(45.3)$ & $351(56.1)$ & $194(31.0)$ & $81(12.9)$ \\
\hline $65-74$ & 494 (35.7) & $122(24.7)$ & $303(61.3)$ & $69(14.0)$ \\
\hline$\geq 75$ & $179(13.0)$ & $14(7.8)$ & $151(84.4)$ & $14(7.8)$ \\
\hline \multicolumn{5}{|l|}{ Income class } \\
\hline $0-2$ & $211(15.3)$ & $68(32.2)$ & $123(58.3)$ & $20(9.5)$ \\
\hline $3-4$ & $140(10.1)$ & $43(30.7)$ & $79(56.4)$ & $18(12.9)$ \\
\hline 5-6 & $211(15.3)$ & $84(39.8)$ & $104(49.3)$ & $23(10.9)$ \\
\hline $7-8$ & $280(20.2)$ & 115 (41.1) & $125(44.6)$ & $40(14.3)$ \\
\hline $9-10$ & $540(39.1)$ & $226(41.8)$ & $238(44.1)$ & $76(14.1)$ \\
\hline \multicolumn{5}{|l|}{ Residential area } \\
\hline Metropolitan & 354 (25.6) & $154(43.5)$ & 147 (41.5) & $53(15.0)$ \\
\hline Urban & $317(22.9)$ & $114(36.0)$ & $156(49.2)$ & $47(14.8)$ \\
\hline Suburban/Rural & $711(51.5)$ & $268(37.7)$ & $366(51.5)$ & $77(10.8)$ \\
\hline
\end{tabular}

$\mathrm{ADT}$, androgen deprivation therapy; RT, radiation therapy.

variables. Annual $p$ for trend was determined by Wilcoxontype test for trend across ordered groups. Multivariable multinomial logistic regression analysis was used to determine the adjusted odds ratios (OR) and 95\% confidence intervals $(\mathrm{CI})$ of sociodemographic factors that showed significant association with treatment choices (based on the surgery group). Potential confounders included age, income class, residential area, and year diagnosed. All tests were two-tailed, with $p<0.05$ considered significant. Statistical analysis was performed using Stata/SE software ver. 12.1 (Stata Corp., College Station, TX).

\section{Results}

\section{Characteristics of the study population}

A summary of the patient sociodemographic factors is shown in Table 1. Mean age at diagnosis was 64.1 years (standard deviation, 9.4 years). There were 83 patients (15.3\%) in the lowest income classes (class 0-2), and the greatest percentage $(39.1 \%)$ was in the highest income classes (class 9-10). Approximately one fourth of patients lived in a metropolitan area, and over $50 \%$ of patients lived in a suburban or rural area. Of the entire study population, patients undergoing surgery, ADT, and RT were $38.8 \%, 48.4 \%$, and $12.8 \%$, respectively.

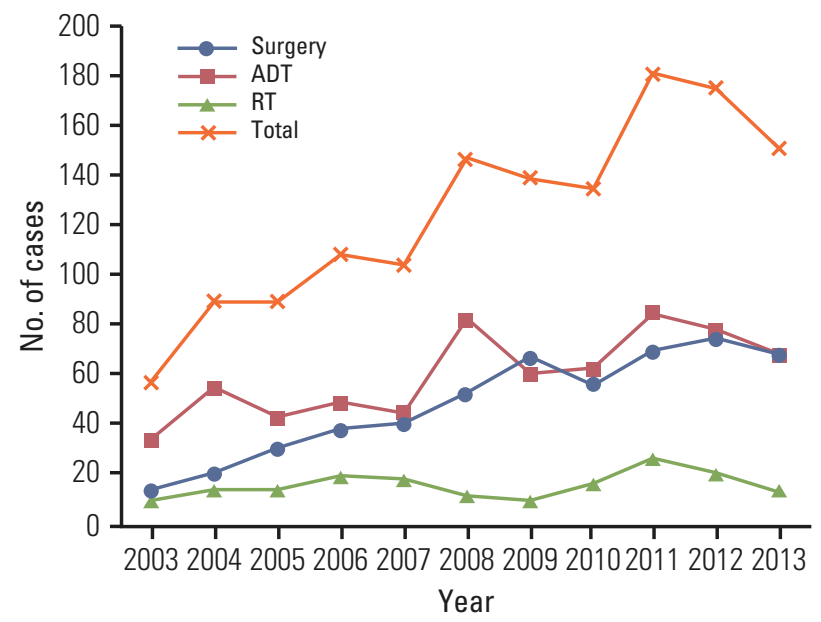

Fig. 1. Trends in primary treatment for prostate cancer in Korea. ADT, androgen deprivation therapy; RT, radiation therapy.

\section{Trends in primary treatment for PC}

Overall, the total number of patients undergoing active treatments for PC showed a significant increase. As shown in Fig. 1, the total number of cases increased from 58 in 2003 to 152 in 2013 , corresponding to a $162 \%$ increase during 10 years. Of the treatment modalities, surgery cases showed the most significant increase, thus relative proportion increased from $22.4 \%$ in 2003 to $45.4 \%$ in 2013 ( $p$ for trend 
A
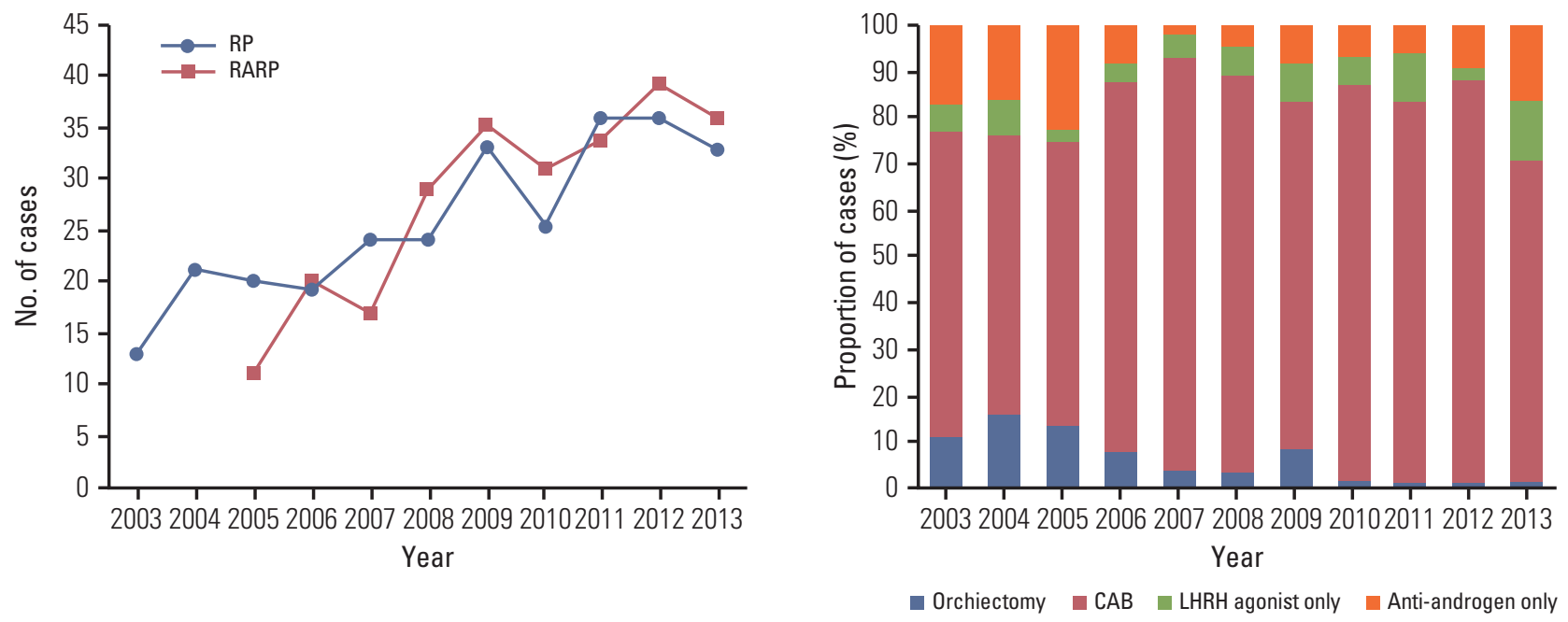

Fig. 2. Trends in radical surgery (A) and androgen deprivation therapy (B) for prostate cancer in Korea. RP, radical prostatectomy; RARP, robot-assisted radical prostatectomy; $\mathrm{CAB}$, combined androgen blockade; $\mathrm{LHRH}$, luteinizing hormonereleasing hormone.

$<0.001$ ). While ADT cases have increased, the relative proportion of ADT showed a tendency to decrease, from $60.3 \%$ in 2003 to $45.4 \%$ in 2013 ( $\mathrm{p}$ for trend $=0.020$ ). While RT cases increased slightly over the 10 years, the relative proportion was variable $(17.2 \%$ in $2003,18.4 \%$ in $2006,7.2 \%$ in 2009 , and $9.2 \%$ in 2013) and the increment of change was relatively small compared to that of surgery and ADT cases ( $\mathrm{p}$ for trend $=0.034$ ).

RARP was introduced in 2005 in many hospitals in Korea. While RP cases have shown a steady increase, RARP cases have shown a more rapid increase, thus RARP cases exceeded RP cases in 2013 (Fig. 2A). Regarding the types of ADT, orchiectomy cases showed a remarkable decrease, from over $12 \%$ in early 2000s to $1.4 \%$ in 2013 (Fig. 2B). CAB was the most commonly used (responsible for more than $62 \%$ to $89 \%$ of ADT), whereas use of LHRH agonists and anti-androgen monotherapy did not show definite trends.

\section{Treatment patterns according to sociodemographic fac- tors}

Treatment patterns differed significantly according to age group ( $\mathrm{p}<0.001$ ) (Fig. 3A) and income class $(\mathrm{p}=0.014)$ (Fig. 3B), respectively. As expected, surgery was the most common primary treatment in patients younger than 64 years, accounting for $59.0 \%$ in < 49 years and $56.1 \%$ in $50-64$ years, respectively (Fig. 3A). In contrast, surgery was performed in only $7.8 \%$ of patients older than 75 years, whereas the majority of those patients $(84.4 \%)$ underwent ADT.
Patients with higher income showed a tendency for definite therapy including surgery and RT, whereas patients with lower income showed a tendency for ADT (Fig. 3B). Treatment patterns according to residential area are shown in Fig. 4. While no definite trends were observed, there were differences in primary treatment according to residential area.

Multivariable multinomial logistic regression analysis was performed to assess independent effects of patient sociodemographic factors on treatment choice. As shown in Table 2, patients older than 65 years showed significant association with ADT compared to surgery (patients aged 65-74 years: adjusted OR, 5.72; 95\% CI, 3.25 to 10.04; patients $\geq 75$ years: adjusted OR, 26.59; 95\% CI, 12.30 to 57.52), while patients older than 75 years showed significant association with RT (adjusted OR, 3.01; 95\% CI, 1.12 to 8.10). Patients with higher income levels showed significant association with surgery compared to ADT (7-8 income class: adjusted OR, 0.59; 95\% CI, 0.38 to 0.90 ; $9-10$ income class: adjusted OR, 0.56 ; $95 \%$ CI, 0.38 to 0.82 ). In addition, patients living in urban areas showed significant association with ADT use compared to surgery (adjusted OR, 1.47; 95\% CI, 1.02 to 2.12). As the year increased, the probability of RT use compared to surgery decreased significantly (adjusted OR, 0.93; 95\% CI, 0.87 to 0.99). 
A
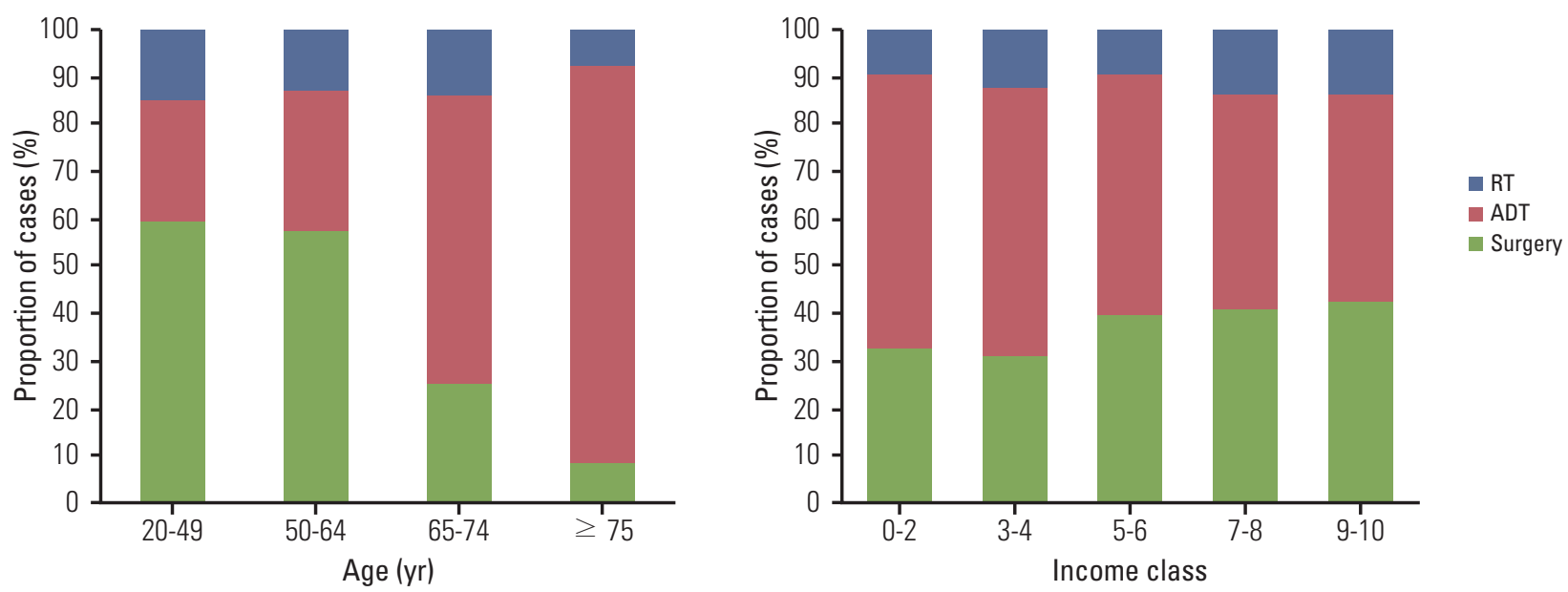

Fig. 3. Treatment patterns according to age group (A) and income class (B). RT, radiation therapy; ADT, androgen deprivation therapy.

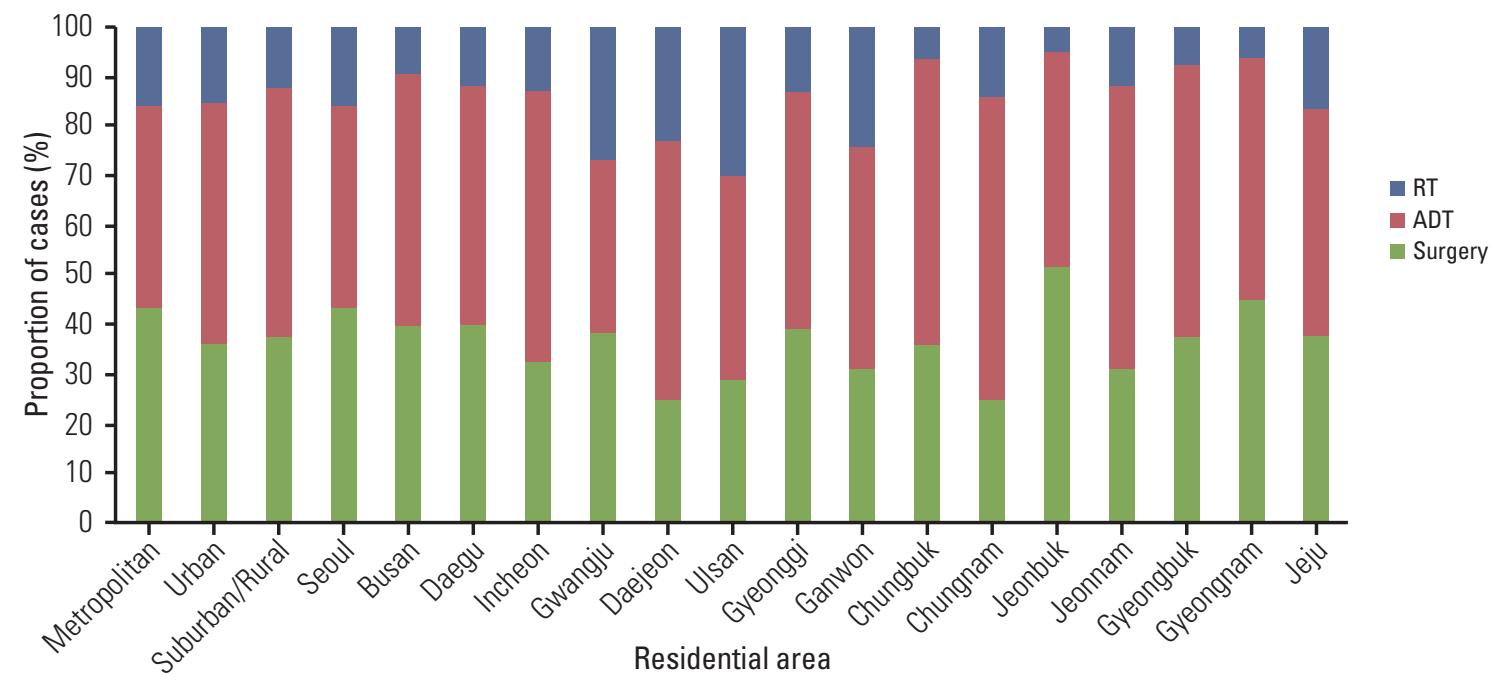

Fig. 4. Treatment patterns according to residential area. RT, radiation therapy; ADT, androgen deprivation therapy.

\section{Discussion}

To the best of our knowledge, this is the first study investigating changing patterns of primary treatment in Korean PC patients over 10 years from a nationwide population based cohort. In line with a rapid increase in the incidence and prevalence of PC in Korean men, the total number of patients undergoing active treatments increased significantly (Fig. 1). Of the treatment modalities, remarkable increase in surgery cases is probably attributable to early detection of PC due to PSA screening. Although clinical benefit of routine PSA screening remains controversial [9,10], PSA screening in Korea has increased with increase of routine health check- 
Table 2. Association of sociodemographic factors with primary treatment modality

\begin{tabular}{|c|c|c|c|c|}
\hline \multirow{2}{*}{ Variable } & \multicolumn{2}{|c|}{ ADT } & \multicolumn{2}{|c|}{ RT } \\
\hline & OR & $95 \%$ CI & OR & $95 \%$ CI \\
\hline \multicolumn{5}{|l|}{ Age (yr) } \\
\hline $20-49$ & 1 & 1 & 1 & 1 \\
\hline $50-64$ & 1.25 & $0.72-2.18$ & 0.82 & $0.42-1.60$ \\
\hline $65-74$ & 5.72 & $3.25-10.04$ & 1.97 & $0.99-3.94$ \\
\hline$\geq 75$ & 26.59 & $12.30-57.52$ & 3.01 & $1.12-8.10$ \\
\hline \multicolumn{5}{|l|}{ Income class } \\
\hline $0-2$ & 1 & 1 & 1 & 1 \\
\hline 3-4 & 1.14 & $0.68-1.92$ & 1.49 & $0.70-3.16$ \\
\hline $5-6$ & 0.75 & $0.48-1.18$ & 0.94 & $0.47-1.88$ \\
\hline $7-8$ & 0.59 & $0.38-0.90$ & 1.09 & $0.58-2.03$ \\
\hline $9-10$ & 0.56 & $0.38-0.82$ & 1.05 & $0.59-1.86$ \\
\hline \multicolumn{5}{|l|}{ Residential area } \\
\hline Metropolitan & 1 & 1 & 1 & 1 \\
\hline Urban & 1.47 & $1.02-2.12$ & 1.17 & $0.73-1.87$ \\
\hline Suburban/Rural & 1.23 & $0.90-1.67$ & 0.77 & $0.51-1.17$ \\
\hline Year (continuous) & 1.01 & $0.96-1.05$ & 0.93 & $0.87-0.99$ \\
\hline
\end{tabular}

Analysis by multinomial logistic regression with outcome variable (treatment modality) based on surgery group. ADT, androgen deprivation therapy; $\mathrm{RT}$, radiation therapy; $\mathrm{OR}$, odds ratio; $\mathrm{CI}$, confidence interval.

up compared to the past [11,12]. Notably, while the number of patients undergoing surgery showed a significant increase, the relative proportion of patients undergoing RT was variable over 10 years (from $7.2 \%$ to $18.4 \%$ ). In western countries, RT is a main treatment modality for radical treatment of PC, thus its use constitutes from $19 \%$ to $41 \%$ for definite therapy $[1,5,13]$. These findings indicate that the main treatment modality for radical treatment in Korean PC patients is surgery, whereas RT is relatively underused. In Korea, the majority of PC patients are diagnosed by urologists, therefore dominant use of surgery as primary definite therapy may in part be attributable to clinician factors. In addition, because most private medical insurance in Korea reimburses only inpatients but does not reimburse outpatients, such economic factors may affect patients' treatment choice. Similar to our finding, only $4.9 \%$ of patients chose RT as primary treatment for PC in a Japanese study [14]. In that study [14], attitude of Japanese patients, who rarely ask for a second opinion from a clinician, was suggested as one possible reason for this finding.

We also assessed trends in surgery and primary ADT. Regarding surgical modalities, we found that use of RARP has increased since its introduction in 2005, and the number of cases showed a remarkable increase in 2008 because the installation of the da Vinci surgical system increased dramatically in 2008, consistent with a recent study [15] which reported an increasing trend of the total number of RARP on the basis of the data from Intuitive Surgical Korea Ltd. (Seoul, Korea). As such, RARP use recently exceeded conventional RP. Existing evidence, including ours, has shown comparable or superior oncological and functional outcomes (incontinence and potency) between RARP and RP [16,17], although several studies reported conflicting results regarding this issue [18]. In addition, besides the high initial cost of purchasing a robot, RARP is not reimbursed by KNHI. Thus economic cost of RARP is significantly higher than that of $R P$. To address the question of whether RARP is more beneficial than RP in terms of cost-effectiveness [19], further critical assessment of the economics involved in the two surgical modalities is necessary.

ADT is still commonly used for treatment of Korean PC patients, accounting for more than $60 \%$ in 2003 and $45 \%$ in 2013 , although its use showed a tendency to decrease. We observed two main findings regarding ADT use in Korea: surgical castration has seldom been used (less than 1.4\%) recently, and the majority (60\% to $88.9 \%$ ) of Korean urologists use CAB for primary ADT. Because KNHI data do not include information regarding clinical stage, we cannot address the proportions of ADT use according to clinical stage. Considering the aforementioned increase of early PC detection, a significant proportion of patients with localized PC may still undergo ADT as primary therapy, which is of uncertain benefit in this setting [20], due to various reasons including patient anxiety [21] and clinician factors [7,22]. 
In a US population-based study using the Surveillance, Epidemiology, and End Results-Medicare linked database [22], urologists with no academic affiliation were significantly more likely to use primary ADT for localized PC compared to urologists with a major academic affiliation. Some (approximately 10\% during the study period) urologists use anti-androgen monotherapy, which is not recommended based on existing evidence [23]. These findings represent variation in ADT use that is affected by clinician practice style in addition to tumor or patient characteristics [7].

Our results clearly show that sociodemographic factors do affect primary treatment choice. We confirmed the impact of each sociodemographic factor (i.e., age, income class, and residential area) on treatment choice even after adjusting other parameters (Table 2). As expected, older patients showed significant association with ADT or RT. Income levels showed significant association with primary treatment. Specifically, patients with higher income showed significant association with surgery. This finding may indicate that patients with higher income are more likely to undergo routine health check-up and be diagnosed with early stage PC, thus being more predisposed to surgery. Similar finding was observed in other studies that reported an inverse relationship between poverty and surgery rates [24,25]. We also observed regional variations in treatment patterns (Fig. 4) and significant association between urban area and ADT use compared to surgery (Table 2). These regional variations may be attributable to differences in the availability of treatment modalities and consequential discrepancies of information given to patients.

We acknowledge several possible limitations of our study. Because data regarding clinical and pathologic stage are not available in the KNHI database, treatment patterns according to stage and multivariable multinomial logistic regression analysis adjusting for stage could not be analyzed. In addition, the proportion of Korean PC patients on watchful waiting or active surveillance, which is a reasonable treatment option in low risk PC patients, could not be determined because those options do not have a KNHI reimbursement code. For a similar reason, RARP was operationally defined, thus the estimated number of RARP cases may be somewhat different from the actual number of cases. Countering the possible limitations, our results are based on a nationwide, randomly selected population based cohort, thus generalizable to the entire Korean PC population. Considering that our estimated number of RARP cases is consistent with the recently published result [15], we believe that our operational definition for RARP appropriately represents an overall increasing trend of RARP in Korea. In addition, analysis of time trends over 10 years highlights changing patterns of primary treatment for PC in Korea. Because our study included only patients who had undergone active treatments for newly diagnosed PC, we believe that confounding effects due to diagnosis coding errors in claims data would be minimal.

\section{Conclusion}

While total cases of active treatments for PC showed a significant increase, the treatment pattern in Korean PC patients has changed remarkably over the last 10 years. The proportion of patients undergoing surgery has increased significantly while the proportion of patients undergoing RT has been relatively constant. In addition, sociodemographic factors do affect the primary treatment choice. Our results will be valuable in overviewing changing patterns of primary treatment in Korean PC patients and planning future health policy for PC.

\section{Conflicts of Interest}

Conflict of interest relevant to this article was not reported.

\section{Acknowledgments}

This study was partly supported by a National Research Foundation of Korea (NRF) Grant funded by the Korean Government (MSIP) (NRF-2015R1A1A1A05001042). This study used National Health Insurance Service (NHIS) National Sample Cohort (NSC) data (NHIS-2014-2-032) made by NHIS. The author(s) declare no conflict of interest with NHIS. 


\section{References}

1. DeSantis CE, Lin CC, Mariotto AB, Siegel RL, Stein KD, Kramer JL, et al. Cancer treatment and survivorship statistics, 2014. CA Cancer J Clin. 2014;64:252-71.

2. Jung KW, Won YJ, Kong HJ, Oh CM, Lee DH, Lee JS. Cancer statistics in Korea: incidence, mortality, survival, and prevalence in 2011. Cancer Res Treat. 2014;46:109-23.

3. Jung KW, Won YJ, Kong HJ, Oh CM, Cho H, Lee DH, et al. Cancer statistics in Korea: incidence, mortality, survival, and prevalence in 2012. Cancer Res Treat. 2015;47:127-41.

4. Lamb DS, Bupha-Intr O, Bethwaite P, Murray J, Nacey J, Russell G, et al. Prostate cancer: are ethnic minorities disadvantaged? Anticancer Res. 2008;28:3891-5.

5. Mettlin CJ, Murphy GP, Cunningham MP, Menck HR. The National Cancer Data Base report on race, age, and region variations in prostate cancer treatment. Cancer. 1997;80: 1261-6.

6. Sadetsky N, Elkin EP, Latini DM, DuChane J, Carroll PR; CaPSURE Investigators. Prostate cancer outcomes among older men: insurance status comparisons results from CaPSURE database. Prostate Cancer Prostatic Dis. 2008;11:280-7.

7. Shahinian VB, Kuo YF, Freeman JL, Goodwin JS. Determinants of androgen deprivation therapy use for prostate cancer: role of the urologist. J Natl Cancer Inst. 2006;98:839-45.

8. Jung KW, Won YJ, Kong HJ, Oh CM, Shin A, Lee JS. Survival of Korean adult cancer patients by stage at diagnosis, 20062010: national cancer registry study. Cancer Res Treat. 2013; 45:162-71.

9. Schroder FH, Hugosson J, Roobol MJ, Tammela TL, Ciatto S, Nelen $\mathrm{V}$, et al. Screening and prostate-cancer mortality in a randomized European study. N Engl J Med. 2009;360:1320-8.

10. Andriole GL, Crawford ED, Grubb RL 3rd, Buys SS, Chia D, Church TR, et al. Mortality results from a randomized prostate-cancer screening trial. N Engl J Med. 2009;360: 1310-9.

11. Yoo DS, Woo SH, Cho S, Kang SH, Kim SJ, Park SY, et al. Practice patterns of urologists in managing Korean men aged 40 years or younger with high serum prostate-specific antigen levels. Urology. 2014;83:1339-43.

12. Park SK, Sakoda LC, Kang D, Chokkalingam AP, Lee E, Shin $\mathrm{HR}$, et al. Rising prostate cancer rates in South Korea. Prostate. 2006;66:1285-91.

13. Cooperberg MR, Broering JM, Carroll PR. Time trends and local variation in primary treatment of localized prostate cancer. J Clin Oncol. 2010;28:1117-23.

14. Nakamura K, Teshima T, Takahashi Y, Imai A, Koizumi M, Mitsuhashi N, et al. Radical radiation therapy for prostate can- cer in Japan: a patterns of care study report. Jpn J Clin Oncol. 2003;33:122-6.

15. Seo IY. Urologic robotic surgery in Korea: past and present. Korean J Urol. 2015;56:546-52.

16. Park J, Yoo DS, Song C, Park S, Park S, Kim SC, et al. Comparison of oncological outcomes between retropubic radical prostatectomy and robot-assisted radical prostatectomy: an analysis stratified by surgical experience. World J Urol. 2014; 32:193-9.

17. Tewari A, Sooriakumaran P, Bloch DA, Seshadri-Kreaden U, Hebert AE, Wiklund P. Positive surgical margin and perioperative complication rates of primary surgical treatments for prostate cancer: a systematic review and meta-analysis comparing retropubic, laparoscopic, and robotic prostatectomy. Eur Urol. 2012;62:1-15.

18. Barry MJ, Gallagher PM, Skinner JS, Fowler FJ Jr. Adverse effects of robotic-assisted laparoscopic versus open retropubic radical prostatectomy among a nationwide random sample of medicare-age men. J Clin Oncol. 2012;30:513-8.

19. Lotan Y, Cadeddu JA, Gettman MT. The new economics of radical prostatectomy: cost comparison of open, laparoscopic and robot assisted techniques. J Urol. 2004;172(4 Pt 1):1431-5.

20. Lu-Yao GL, Albertsen PC, Moore DF, Shih W, Lin Y, DiPaola RS, et al. Survival following primary androgen deprivation therapy among men with localized prostate cancer. JAMA. 2008;300:173-81.

21. Dale W, Hemmerich J, Bylow K, Mohile S, Mullaney M, Stadler WM. Patient anxiety about prostate cancer independently predicts early initiation of androgen deprivation therapy for biochemical cancer recurrence in older men: a prospective cohort study. J Clin Oncol. 2009;27:1557-63.

22. Shahinian VB, Kuo YF, Freeman JL, Orihuela E, Goodwin JS. Characteristics of urologists predict the use of androgen deprivation therapy for prostate cancer. J Clin Oncol. 2007;25: 5359-65.

23. Heidenreich A, Bastian PJ, Bellmunt J, Bolla M, Joniau S, van der Kwast T, et al. EAU guidelines on prostate cancer. Part II: Treatment of advanced, relapsing, and castration-resistant prostate cancer. Eur Urol. 2014;65:467-79.

24. Krupski TL, Kwan L, Afifi AA, Litwin MS. Geographic and socioeconomic variation in the treatment of prostate cancer. J Clin Oncol. 2005;23:7881-8.

25. Polednak AP. Prostate cancer treatment in black and white men: the need to consider both stage at diagnosis and socioeconomic status. J Natl Med Assoc. 1998;90:101-4. 